\title{
Performance of Jets and Missing Transverse Energy in CMS
}

\section{Joram Berger*}

On behalf of the CMS Collaboration

Karlsruhe Institute of Technology

E-mail: joram.bergerecern.ch

\begin{abstract}
A summary of the measurements of jet energy calibration and missing transverse energy calibration and resolution is presented. The jet energy calibration is performed with data samples collected in proton-proton collisions at a centre-of-mass energy of $8 \mathrm{TeV}$ corresponding to an integrated luminosity of $1.6 \mathrm{fb}^{-1}$. The final jet energy calibration is based on dijet, $\gamma+$ jet and $\mathrm{Z}+\mathrm{jet}$ events. The results are presented for the "Particle Flow" approach, which attempts to reconstruct all stable particles in the event individually, prior to the jet clustering, based on information from all relevant subdetectors. We discuss the effect of pile-up interactions and the state of the art mitigation techniques used in CMS. Furthermore, we describe the main uncertainty sources for the jet energy calibration. Finally, the results of comprehensive studies of the missing transverse energy are presented, performed with $0.7 \mathrm{fb}^{-1}$ of $\mathrm{Z}(\rightarrow \mu \mu)+$ jets events collected at $8 \mathrm{TeV}$.
\end{abstract}

36th International Conference on High Energy Physics

4-11 July 2012

Melbourne, Australia

${ }^{*}$ Speaker. 


\section{Reconstruction of Jets and Missing Transverse Energy in CMS}

CMS has achieved a thorough understanding of jets and missing transverse energy $E_{\mathrm{T}}^{\mathrm{miss}}$ on a time scale that has never been reached before. The increased number of pile-up interactions and the mitigation of their effects is the major challenge in 2012.

Within the CMS Collaboration, the "Particle Flow" approach [1] is used for the event reconstruction. It combines information from all detector components prior to jet clustering, $E_{\mathrm{T}}^{\text {miss }}$ calculation and further processing. This yields a higher response and an improved resolution compared to purely calorimeter-based jet and $E_{\mathrm{T}}^{\mathrm{miss}}$ reconstruction. Furthermore, it allows to distinguish contributions to the reconstructed jet energy from different detector components.

\section{Jet Energy Corrections}

The CMS Collaboration uses a factorised approach for jet calibration to combine the advantages of Monte-Carlo simulation based studies with robust data-driven methods. The procedure is described in detail in [2]. The correction levels are:

- corrections for pile-up and electronic noise;

- corrections for detector calibration effects and reconstruction efficiencies based on MonteCarlo simulation;

- corrections for residual dependencies on the pseudorapidity $\eta$ and transverse momentum $p_{\mathrm{T}}$ directly derived from data.

\subsection{Pile-Up Corrections}

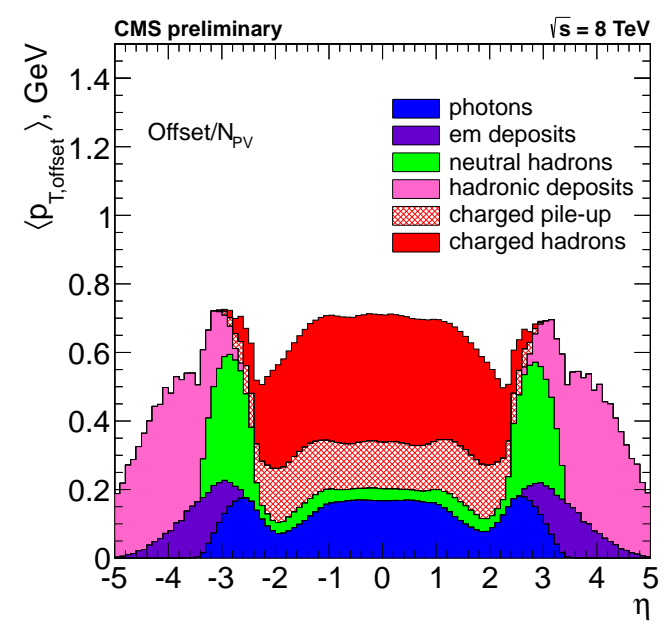

a)

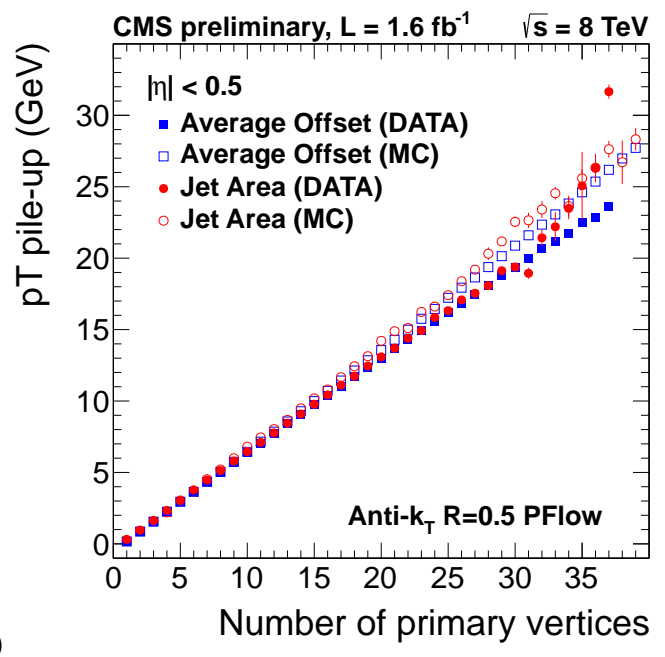

b)

Figure 1: Pile-up corrections: a) Average $p_{\mathrm{T}}^{\text {offset }}$ due to pile-up as a function of $\eta$, broken down to contributions from different components as given by the Particle Flow reconstruction algorithm. b) The pile-up contribution to the jet $p_{\mathrm{T}}$ rises linearly with the number of primary vertices in the event [5].

Dedicated corrections are defined to account for the effects of multiple proton collisions in the same bunch crossing (pile-up). Two methods are used in CMS: the first subtracts from a jet 
the transverse momentum emerging from pile-up as a function the number of primary vertices $N_{\mathrm{PV}}$ reconstructed in the event. The second method extends this approach by taking into account the jet area of the considered jet and the amount of transverse momentum per unit area added to the event by pile-up [4]. The latter is the default method for Particle Flow jets.

\subsection{Corrections Based on MC Simulation}
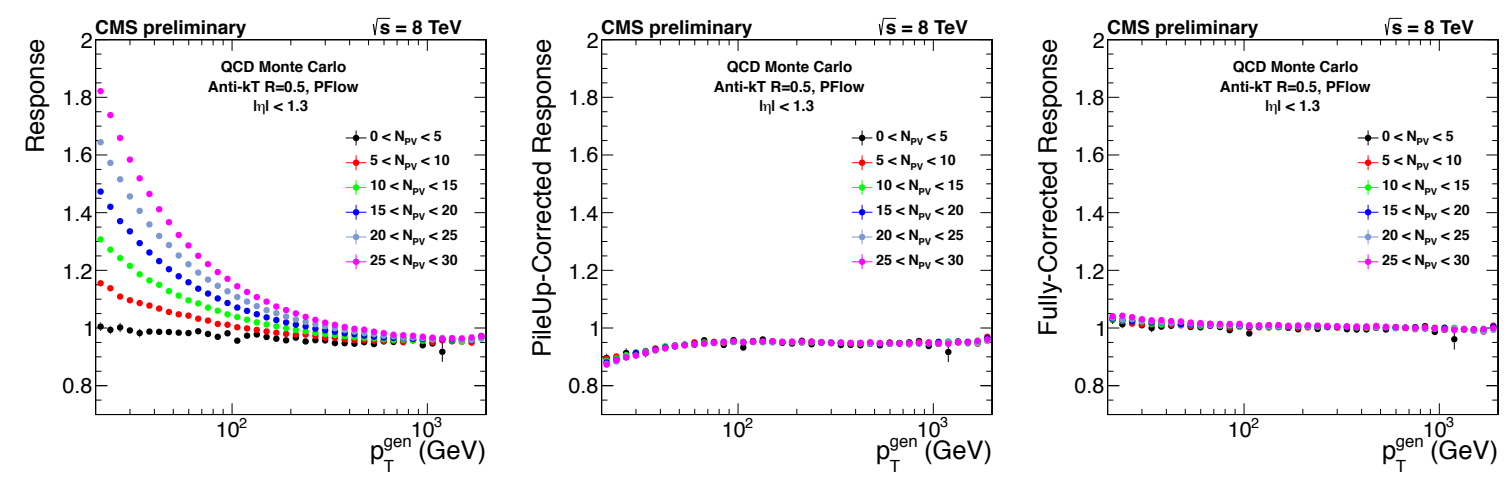

Figure 2: MC based corrections (from left to right): Uncorrected jets show a clear dependence on the number of primary vertices $N_{\mathrm{PV}}$, especially at low $p_{\mathrm{T}}$. This is successfully removed by the pile-up corrections. The corrections to the generator jet show closure over the whole $p_{\mathrm{T}}$ range [5].

After being corrected for the effect of pile-up, jets are corrected to the generator level based on Monte-Carlo simulation of QCD events. This correction step accounts for reconstruction efficiencies and the limited detector resolution. The jet energy scale for various jet flavours differs only by up to $3 \%$ in the central part of the detector. Therefore, flavour corrections are not applied by default. The corrected response reaches unity in simulated events over the whole $p_{\mathrm{T}}$ and $\eta$ range, as is shown in Fig. 2 for jets in the barrel region $(|\eta|<1.3)$.

\subsection{Data-Driven Residual Corrections}

This chain of corrections is completed by the residual corrections in data. Relative differences in the jet energy scale between jets in the central part of the detector and jets with arbitrary $\eta$ are determined from dijet events in collision data. The jets are then corrected to the absolute jet energy scale which is measured in topologies where a well-calibrated reference object is balanced to a jet. Measurements in three different channels are combined: $\mathrm{Z}(\rightarrow \mu \mu)+\mathrm{jet}, \mathrm{Z}(\rightarrow \mathrm{ee})+\mathrm{jet}$ and $\gamma+$ jet. Events of those topologies are selected by requiring additional jets besides the balanced jet to have small transverse momenta compared to the hard scattering scale of the event. Extrapolation techniques are employed to study the ideal case of no additional jet activity in the event. As Fig. 3 depicts, residual corrections are especially needed in the forward region of the detector. The different channels in the determination of the absolute jet energy scale agree within uncertainties.

\subsection{Uncertainties of the Jet Energy Corrections}

The uncertainties on the transverse momentum of a corrected jet strongly depends on its $p_{\mathrm{T}}$ and $\eta$ (Fig. 4). The uncertainty resulting from the difficulty to distinguish pile-up interactions from the hard process affects especially low energy jets, whereas statistical uncertainties due to trigger 


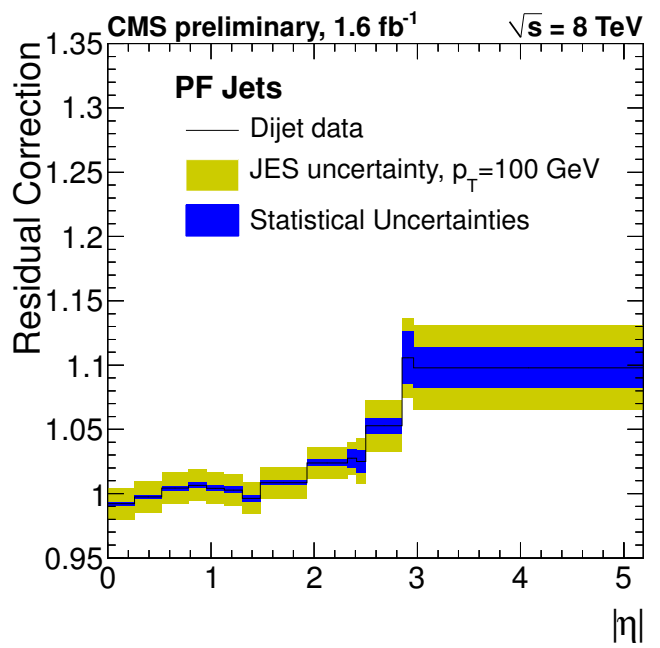

a)

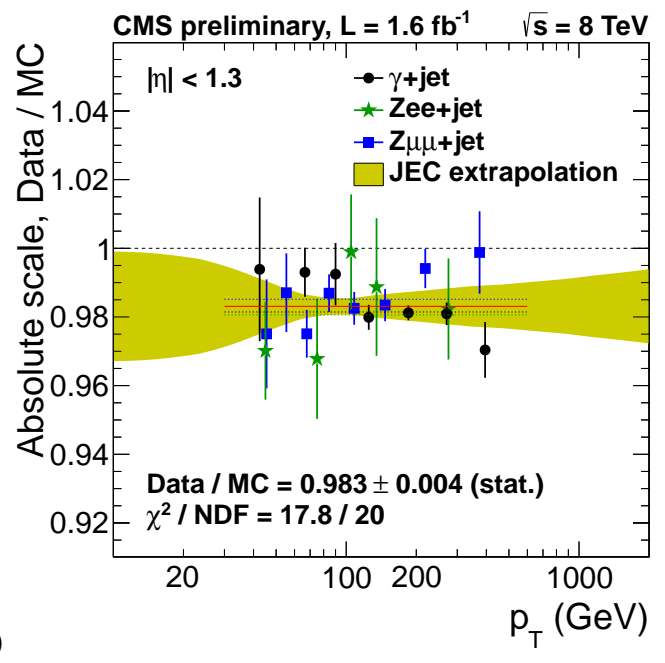

Figure 3: Residual corrections: a) Residual corrections are particularly needed in the forward region. b) The absolute scale in data is slightly lower in data which leads to residual corrections of the absolute $p_{\mathrm{T}}$ [5].
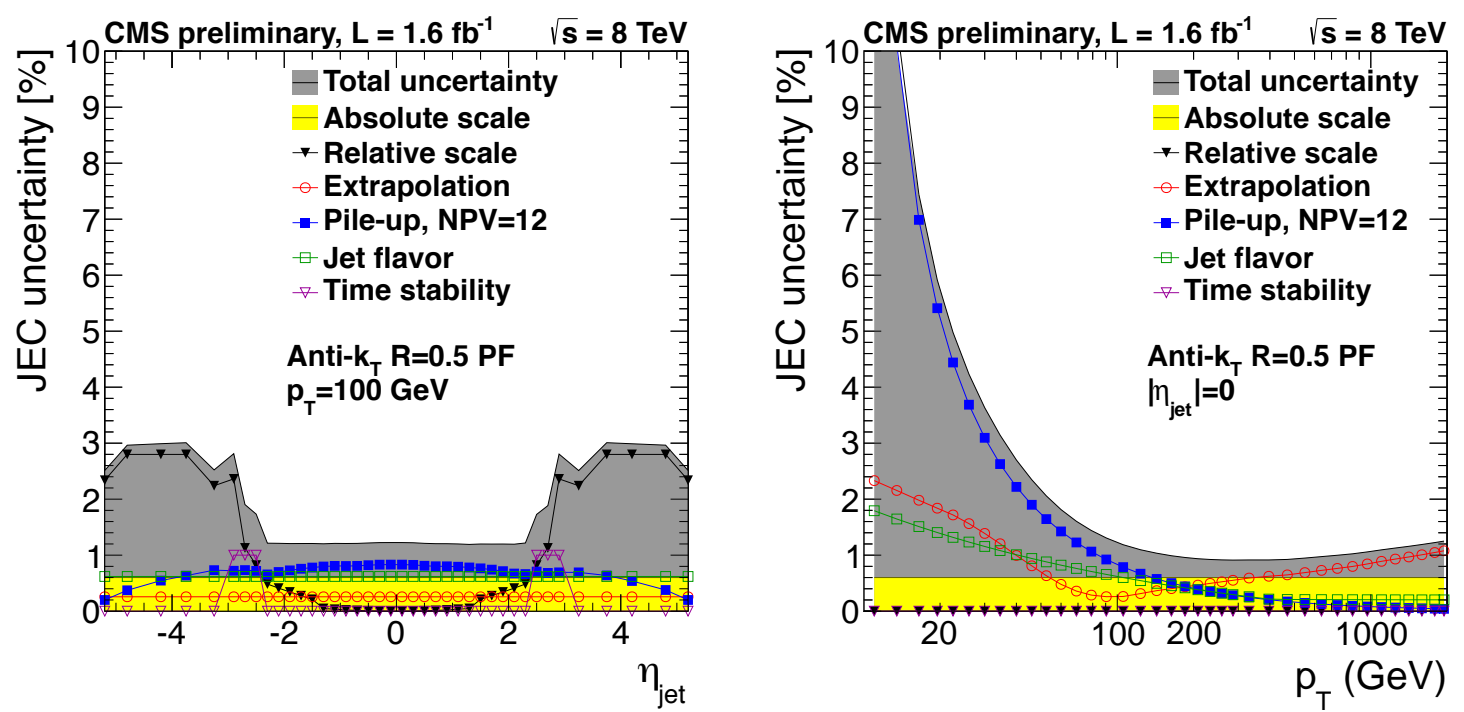

Figure 4: Uncertainty of the final jet energy corrections as a function of $p_{\mathrm{T}}$ (left) and $\eta$ (right) split up into the considered sources of systematic uncertainties [5].

settings and systematic uncertainties of the relative corrections limit the precision in the forward region. In the central part of the detector, the jet energy scale is determined with a precision of about $1 \%$.

\section{Missing Transverse Energy}

Robust missing transverse energy calibration and validation methods have been developed and established in CMS [3]. In 2012, the measurements are performed with $\mathrm{Z}(\rightarrow \mu \mu)$ events [6], taking advantage of the absence of intrinsic $E_{\mathrm{T}}^{\text {miss }}$ and the very high resolution of CMS in the reconstruction of muon four-momenta. Considering the uncertainties, the distribution of the missing 


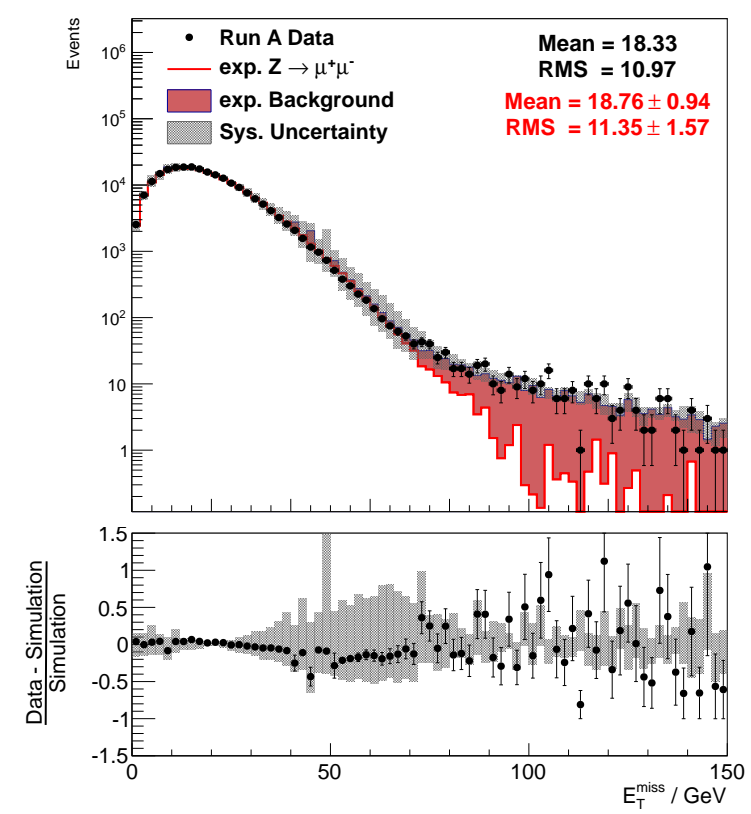

Figure 5: The distribution of the missing transverse energy $E_{\mathrm{T}}^{\text {miss }}$ in data agrees with the expectations from Monte-Carlo simulation [6].

transverse energy in data agrees with the expectations from Monte-Carlo simulation over the whole $p_{\mathrm{T}}$ range, as shown in Fig. 5.

The projection of the transverse momentum of the hadronic recoil $u$ on the one of the $\mathrm{Z}$ boson $q_{\mathrm{T}}$ is used as a measure for the $E_{\mathrm{T}}^{\text {miss }}$ scale. The $E_{\mathrm{T}}^{\text {miss }}$ resolution is determined as the standard deviation of the perpendicular projection of the hadronic recoil to the $\mathrm{Z}$ transverse momentum.

$$
E_{\mathrm{T}}^{\text {miss }} \text { scale }=\left\langle-\frac{u_{\|}}{q_{\mathrm{T}}}\right\rangle \quad E_{\mathrm{T}}^{\text {miss }} \text { resolution }=\sigma\left(u_{\perp}\right)
$$

Compared to previous data taking periods, an improved resolution for a fixed number of primary vertices was achieved in 2012 by the use of dedicated pile-up corrections. Furthermore, improved ECAL and HCAL reconstruction techniques have reduced the impact of pile-up from preceding and following bunch crossings on the $E_{\mathrm{T}}^{\text {miss }}$ measurements. The $E_{\mathrm{T}}^{\text {miss }}$ resolution degradation per additional primary vertex only amounts to $3 \mathrm{GeV}$. The $E_{\mathrm{T}}^{\text {miss }}$ resolution as a function of the number of primary vertices is shown in Fig. 6a.

There are ongoing efforts to reduce the effect of pile-up on the reconstructed missing energy. However, already with these results, CMS can keep up with the increasing luminosity during the 2012 data taking period.

The correction applied to jets can be propagated to $E_{\mathrm{T}}^{\text {miss }}$ to restore the consistency of the event. As Fig. 6b shows, this corrected $E_{\mathrm{T}}^{\text {miss }}$ response is very close to unity over a wide $p_{\mathrm{T}}$ range and the results in data agree with Monte-Carlo simulation. 
a)

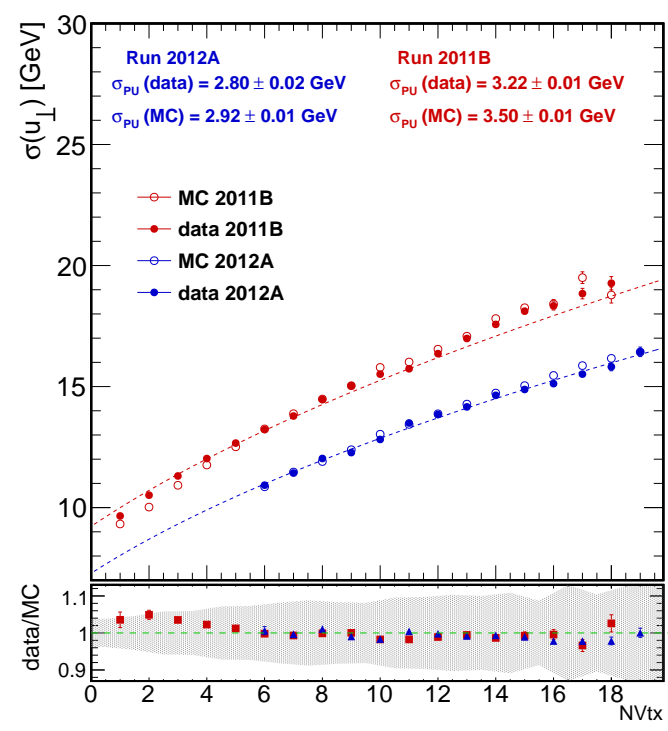

b)

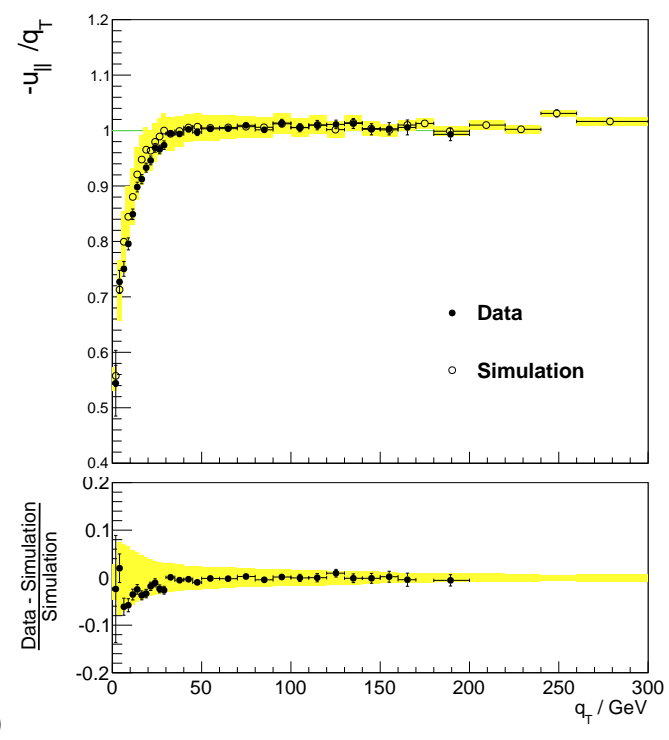

Figure 6: a) $E_{\mathrm{T}}^{\text {miss }}$ resolution $\sigma\left(u_{\perp}\right)$ as a function of the number of primary vertices $N_{\mathrm{Vtx}}$ and b) the corrected $E_{\mathrm{T}}^{\text {miss }}$ response $-\frac{u_{\|}}{q_{\mathrm{T}}}$ as a function of transverse momentum of the $\mathrm{Z}$ boson $q_{\mathrm{T}}[6]$.

\section{Conclusion}

The CMS Collaboration successfully uses a factorised approach for jet energy corrections. Jets are measured with a precision as good as $1 \%$ in CMS, thanks to the extensive use of robust datadriven techniques. The missing transverse energy is well understood and calibrated. The effects of additional proton-proton collisions in the event are successfully removed from jets and missing energy.

\section{References}

[1] CMS Collaboration, Commissioning of the Particle Flow Event Reconstruction with the First LHC Collisions Recorded in the CMS Detector, CMS-PAS-PFT-10-001 (2010).

[2] CMS Collaboration, Determination of Jet Energy Calibration and Transverse Momentum Resolution in CMS, JINST 6 (2011) P11002 (arXiv:1107.4277).

[3] CMS Collaboration, Missing Transverse Energy Performance of the CMS Detector, JINST 6 (2011) P09001 (arXiv:1106.5048).

[4] M. Cacciari, G. P. Salam Pileup Subtraction Using Jet Areas, Phys.Lett.B659, 119-126, 2008 (arXiv:0707.1378).

[5] CMS Collaboration, Jet Energy Corrections and Uncertainties. Detector Performance Plots for 2012, CMS-DP-2012-012.

[6] CMS Collaboration, MET Performance in 2012 Run A CMS Data, CMS-DP-2012-013. 